\title{
Clinical Perspectives on the Use of Different Peripheral Intravenous Catheters with the Implementation of Volume-based Procurement in China
}

\author{
Jian Ming ${ }^{1,2}$, Hsing Jung Li ${ }^{1}$, Yu Wang ${ }^{1}$, Yunfan Ge ${ }^{3}$, Jun Liu ${ }^{1}$, Shanlian $\mathrm{Hu}^{4,}$ * \\ ${ }^{1}$ Real World Solutions, IQVIA China, Shanghai, China \\ ${ }^{2}$ Key Lab of Health Technology Assessment, National Health Commission, School of Public Health, Fudan University, Shanghai, China \\ ${ }^{3}$ Primary Intelligence, IQVIA China, Shanghai, China \\ ${ }^{4}$ School of Public Health, Fudan University, Shanghai, China
}

Email address:

hushanlian@hotmail.com (Shanlian Hu)

${ }^{*}$ Corresponding author

\section{To cite this article:}

Jian Ming, Hsing Jung Li, Yu Wang, Yunfan Ge, Jun Liu, Shanlian Hu. Clinical Perspectives on the Use of Different Peripheral Intravenous Catheters with the Implementation of Volume-based Procurement in China. Science Journal of Public Health. Vol. 9, No. 2, 2021 , pp. $49-56$. doi: $10.11648 /$ j.sjph.20210902.13

Received: February 27, 2021; Accepted: March 12, 2021; Published: March 26, 2021

\begin{abstract}
China introduced a "volume-based procurement (VoBP)" policy for medical products to contain healthcare costs and improve patient access to cost-effective products recently. The VoBP of peripheral intravenous catheters (PIVCs) was piloted in Jiangsu, Shandong and Jiangxi provinces in late 2019. The bidding of PIVCs mainly focused on their prices, while the evaluation standard on clinical performance of PIVCs was less emphasized. In this study, we aim to understand the clinical impacts associated with the utilization of different PIVCs influenced by the implementation of VoBP policy in China. Semistructured interviews were conducted with nurses in Nanjing and Linyi, the pilot cities implementing VoBP. A total of 27 nurses who used a leading multinational corporation's (MNC's) PIVCs before VoBP and changed to China local brands' PIVCs after VoBP were included as informants. In the first 15 interviews, all domains (PIVC placement, complications, product issues, and training) and corresponding concepts listed in conceptual framework were mentioned, indicating that sample size was sufficient. After the change from the MNC's PIVCs to local brands, PIVCs used per patient increased from 2.0 to 3.5 and the average indwelling time decreased from 3.7 days to 2.1 days. Compared with the MNC's PIVCs, those from local brands were associated with a 0.9-minute increase in PIVC insertion time and a 9\% relative decrease in first stick success rate. Furthermore, increased complication rate, quality issues and hard catheter materials, and reduced product training after PIVC change were also mentioned by nurses. This study discloses that PIVC product change following the implementation of VoBP may result in unexpected issues. Therefore, establishing quality evaluation criteria and strengthening product monitoring mechanism are recommended for VoBP policy optimization on medical consumables.
\end{abstract}

Keywords: Peripheral Intravenous Catheters, Volume-Based Procurement, Clinical Performance, Qualitative Research, China

\section{Introduction}

Peripheral intravenous catheters (PIVCs), with an annual sales volume of 2 billion worldwide [1,2], are the most commonly used intravenous (IV) device among inpatients for the administration of medications, fluids, blood products as well as blood sampling [3]. In Europe, PIVCs compose 80-95\% of IV devices used by inpatients [4], while in China, $99 \%$ of tertiary hospitals utilize PIVCs during IV treatments [5].

In June 2018, China's Deepening Reform Commission adopted a novel procurement scheme "National Pilot Plan of Centralized Drug Procurement", a volume-based procurement (VoBP) policy [6]. The goal of VoBP is to contain healthcare costs and improve patient access to costeffective products [7]. With the continuous advancement of 
VoBP, its scope has expanded from drugs-only to high-value medical consumables as well, and a policy document on "Reform Plan of High-value Medical Consumables Management" was published in May 2019. Immediately after the announcement, the State Council approved "Opinions on Deepening the Reform of the Medical Security System" at the end of 2019, which encouraged the VoBP of low-value consumables [8]. Listed in the scope of low-value consumables, the VoBP of PIVC was piloted in Jiangsu, Shandong and Jiangxi provinces in late 2019.

The bidding of PIVCs in the implementation of the VoBP mainly focused on their prices, while the evaluation standard on product performance and clinical use of PIVCs was less emphasized [9, 10]. When evaluating drugs, consistency quality evaluation methods can be applied to confirm pharmaceutical equivalence and bioequivalence and therefore, ensure similar clinical effects [11]. However, similar methods currently cannot be used for medical consumables because of the wide variation in product designs. Additionally, the outcomes of using different medical consumables were decided by not only product design but also user experience and skills. For example, PIVCs, covering a broad category, were differed significantly in product design and quality, which can further influence user experience and patients' outcomes. Poor quality of PIVC or improper insertions can lead to catheter-related complications [12] which may lead to inferior patient experience and additional burden to the healthcare system [13-15].

With the implementation of VoBP, it is important to understand the clinical use of different PIVC products in order to procure those with satisfying clinical outcomes. Nevertheless, the clinical impact of different PIVC products has not been explored so far. Nurses are primary users of PIVC; thus, understanding their perspectives in terms of PIVC use is essential. Based on the above rationale, experienced nurses were interviewed in this research to understand the clinical impact in terms of PIVC placement, catheter-related complications, product quality issues and product training before and after VoBP.

\section{Methods}

\subsection{Qualitative Interviews and Nurse Recruitment}

Semi-structured interviews were conducted among nurses from clinical settings in Nanjing (Jiangsu Province) and Linyi (Shandong Province), the pilot cities implementing VoBP. The researchers of this study conducted investigation and recruited interviewees as an independent third-party to ensure reliability. Purposive sampling [16] was used to recruit senior nurses with at least 5 years of clinical practice experience. Senior nurses were enrolled to ensure that interviewees had enough experience in PIVC insertion and could summarize the frequency of complications that happened in the whole department and supervise product training. In addition to clinical experience, nurses eligible for this study are those who switched to local brands' PIVCs from a leading multinational corporation's (MNC's) PIVCs after the implementation of VoBP, and had more than 6 months of experience in using both the MNC's PIVCs and local PIVCs.

\subsection{Data Collection}

This study was designed with a post-policy improvement purpose; we conducted interviews with nurses who had experienced the implementation of VoBP and had them recall the clinical utilization of PIVCs before VoBP. Qualitative interviews were conducted in Chinese by a trained interviewer between March and April 2020. Interviews were an hour each by phone.

Previous study showed that 3 months was the ideal training time for nurses to learn clinical operations including PIVC insertion when their operation scores were close to stable [17]. Considering the insertion techniques of local PIVC are similar to the MNC's PIVC, the time taken for nurses to get used to local PIVCs should be less than 3 months. In order to avoid the bias caused by unskilled operation during the study period, the regions investigated were where the VoBP on PIVC were implemented for more than 6 months.

An interview guide was developed to solicit nurses' responses regarding the performance of PIVC products before and after VoBP. Four main concepts were included in the interview guide: 1) PIVC placement which included PIVC utilization per patient, insertion time, first stick success rate and indwelling time; 2) product-specific issues to understand different PIVC products quality and/or design problems; 3) PIVC-related complications including redness, pain or swelling, phlebitis, occlusion, catheter displacement, extravasation and catheter-related bloodstream infection (CRBSI); and 4) product training to investigate perceived benefits of training as well as training frequency and duration. The discussion guide was provided to all interviewees one week prior to the interview to ensure that participants had enough time to summarize and recall the PIVC use information.

Additionally, to quantify the complication rates, nurses were specifically asked for the number of patients using PIVCs from the MNC's PIVC one month before VoBP and using local PIVC one month before the interview, and the number of patients with complications in each circumstance. Complication data were extracted and extrapolated to annual rates. Regarding the number of PIVC use per patient, interviewees were asked "transfusion days per patient", "PIVC indwelling time per patient" and "number of PIVC use per patient" and then the interviewer cross-checked the data points during interviews (number of PIVC use is equal to transfusion days divided by PIVC indwelling time) to ensure the data accuracy. The calculation formulas for complication rate and the number of PIVC use per patient are illustrated below. Interviews were digitally recorded, anonymized and transcribed. Verbatim transcription was applied during the transcribing process. The analysis was conducted in Chinese and then translated into English. 


$$
\begin{aligned}
& \text { Complication rate }=\frac{\text { Number of patients with a certain complication }}{\text { number of patients using intravenous catheters }} \\
& \text { Number of PIVC use per patient }=\frac{\text { Average time of hospital stay }}{\text { Average indwelling time of PIVC }}
\end{aligned}
$$

Notes: The number is based on the scenario in a certain month (the last month of using MNC PIVC before the VoBP or the last month of using local PIVC before the interview); The number is extracted from the interview.

\subsection{Data Analysis}

The data analysis was conducted in two stages: a coding stage following by a summarizing stage. In the coding stage, two researchers with graduate-level training in coding, independently labeled relevant words, phrases or sentences. Specifically, researchers noted answers that were relevant to preconceived concepts, were repeated and/or emphasized by nurses, or were unforeseen. Any disagreements were resolved by consensus and if a consensus could not be met a third researcher arbitrated. In the summarization stage, two researchers reviewed all the identified codes and independently created categories for similar concepts and developed conceptual frameworks. Inconsistencies were resolved by discussion between the two researchers and a third researcher to reach consensus. The final conceptual framework was used to systematically organize the defined concepts and corresponding domains. The corresponding quotations from the nurses (translated from Chinese language to English language) were also extracted to further support concepts and domains. For domains containing quantitative results, mean and relative differences were calculated to quantify the results of clinical performance.

To ensure content validity, concepts were checked for saturation. Data saturation is reached when there is enough information to replicate the study $[18,19]$, and when no new information is available from additional interview research [20]. Failure to reach data saturation has an impact on the quality of research and hampers content validity [21, 22]. To check for saturation, the frequency of concepts was recorded in a saturation grid, and the sample size was considered sufficient with saturation point reached when no new concepts or themes were introduced with additional interviews [23-25].

\subsection{Ethics Statement}

Since this was a qualitative study targeting at nurses with the objective to evaluate the performance of different PIVCs, the study did not collect any health-related information of interviewees. Interviewees were only asked about hospital level, department, title and working experience in order to check their eligibility. Interviewees voluntarily decided whether they were willing to participate in this study and all interviewees provided signed consent forms prior to their participation. In addition, the interviews were anonymous, and this study only reported summarized data; thus, nurses could not be identified. With the above considerations, the study did not seek additional approval from an Independent Ethics Committee or Institutional Review Board.

\section{Results}

\subsection{Study Sample}

Among the 79 nurses we contacted, 27 of them accepted the interview. A total of 27 nurses from 16 clinical settings in Nanjing and Linyi, as informants, were included in this study. All the nurses interviewed are female. Most enrolled nurses were from tertiary hospitals and $93 \%$ had at least 10 years of work experience. Additionally, $44 \%$ and $56 \%$ of interviewees were senior nurses and chief nurses, respectively. Most nurses were from Internal Medicine (37\%), followed by Pediatric (22\%) and Oncology (19\%) departments. Detailed characteristics of the interviewees are provided in Table 1. All 27 nurses used exclusively the MNC's PIVC before VoBP and changed to local brands, including 2 nurses using Brand D, 4 using Brand F, 14 using Brand $\mathrm{S}$, and 7 using Brand $\mathrm{Y}$ after VoBP.

Table 1. Characteristics of interviewees.

\begin{tabular}{llcc}
\hline Demographic & & N & \% \\
\hline Total & & 27 & 100 \\
Gender & Female & 27 & 100 \\
& $5-9$ & 2 & 7 \\
Work & $10-14$ & 4 & 15 \\
Experience & $15-19$ & 10 & 37 \\
& $>=20$ & 11 & 41 \\
Province/City & Jiangsu/Nanjing & 13 & 48 \\
& Shandong/Linyi & 14 & 52 \\
Hospital Level & Tertiary & 25 & 93 \\
& Secondary & 2 & 7 \\
& Internal Medicine (Without Oncology) & 10 & 37 \\
& Surgery & 3 & 11 \\
& Pediatrics/Neonatology & 6 & 22 \\
Department & Oncology & 5 & 19 \\
& Emergency & 3 & 11 \\
& Senior Nurse & 12 & 44 \\
& Chief nurse & 15 & 56 \\
\hline
\end{tabular}

\subsection{Conceptual Framework and Saturation Grid}

Twenty-four domains were identified under the 4 main concepts of PIVC placement, catheter-related complications, product quality and defects and product training (Table 2). All domains were mentioned in the first 15 interviews and no new concepts/themes appeared in the remaining interviews indicating that saturation was achieved. 
Table 2. Conceptual Framework and Saturation Grid.

\begin{tabular}{|c|c|c|c|c|c|c|c|}
\hline Concept & Domain & $\begin{array}{c}\text { Nurse } \\
1-5 \\
\end{array}$ & $\begin{array}{c}\text { Nurse } \\
6-10 \\
\end{array}$ & $\begin{array}{c}\text { Nurse } \\
11-15 \\
\end{array}$ & $\begin{array}{c}\text { Nurse } \\
16-20 \\
\end{array}$ & $\begin{array}{c}\text { Nurse } \\
21-27 \\
\end{array}$ & $\begin{array}{c}\text { Total } \\
\text { Counts } \\
\end{array}$ \\
\hline & Increase in the number of PIVC use per patient & $4 *$ & 3 & 5 & 5 & 6 & 23 \\
\hline PIVC & Increase in PIVC insertion time & $3 *$ & 2 & 2 & 3 & 3 & 13 \\
\hline \multirow[t]{4}{*}{ Placement } & Decrease in first stick success rate & $2 *$ & 4 & 3 & 5 & 5 & 19 \\
\hline & Decrease in indwelling time of PIVC & $4 *$ & 5 & 5 & 5 & 7 & 26 \\
\hline & Increase in redness at insertion site & $3 *$ & 3 & 5 & 4 & 7 & 22 \\
\hline & Increase in swelling/ complaint about pain & $2 *$ & 4 & 5 & 5 & 6 & 22 \\
\hline \multirow{7}{*}{$\begin{array}{l}\text { Catheter- } \\
\text { related } \\
\text { Complications }\end{array}$} & Increase in occlusion & $2 *$ & 4 & 5 & 5 & 7 & 23 \\
\hline & Increase in catheter displacement & $2 *$ & 2 & 3 & 2 & 2 & 11 \\
\hline & Increase in extravasation & 0 & $2 *$ & 4 & 4 & 6 & 16 \\
\hline & Increase in catheter-related bloodstream infection & 0 & 0 & $1 *$ & 0 & 0 & 1 \\
\hline & Flimsy needle (difficult to puncture and easy to bend) & $2 *$ & 5 & 2 & 3 & 3 & 15 \\
\hline & Hard catheter (lead to patient's uncomfortable experience) & $4 *$ & 1 & 1 & 2 & 5 & 13 \\
\hline & Kinking of the connection catheter & 0 & $1 *$ & 0 & 0 & 0 & 1 \\
\hline Product Issues & Poor fit of steel needle and soft needle & 0 & $2 *$ & 1 & 0 & 0 & 3 \\
\hline & Unsmooth pulley & $1 *$ & 0 & 0 & 0 & 0 & 1 \\
\hline & Lengthy needle (not convenient for single-handed operation) & $2 *$ & 2 & 0 & 0 & 1 & 5 \\
\hline & Lengthy heparin cap \# (increase return blood volume and cause blocked catheter) & $1 *$ & 0 & 1 & 1 & 1 & 4 \\
\hline \multirow{5}{*}{$\begin{array}{l}\text { Product } \\
\text { Training }\end{array}$} & Decrease in training frequency & $3 *$ & 2 & 3 & 1 & 2 & 11 \\
\hline & Decrease in training duration & $1 *$ & 2 & 2 & 0 & 0 & 5 \\
\hline & Decrease in training quality & $3 *$ & 3 & 0 & 2 & 2 & 10 \\
\hline & Increase nurses' confidence & $1 *$ & 0 & 0 & 0 & 0 & 1 \\
\hline & Benefit clinical operation & $4 *$ & 4 & 2 & 0 & 3 & 13 \\
\hline
\end{tabular}

*Indicates that this group was the first of the five groups in which a new concept was reported spontaneously. No new concepts/themes appeared in the last two groups, indicating the study reached the saturation.

\# The length of the heparin cap is longer for local PIVC compared with the MNC's PIVC, resulting in more blood return at the time of drawing the blood, increased flush time as well as increased risk of catheter blocking.

Table 3. The relative difference in PIVC placement, complications, and product training between the MNC's PIVC and local PIVC.

\begin{tabular}{|c|c|c|c|c|}
\hline Concept & Indicator & MNC's PIVC (Mean) & Local PIVC (Mean) & Relative Difference \\
\hline \multirow{4}{*}{ PIVC Placement } & Number of PIVC use per patient & 2.0 & 3.5 & $+75 \%$ \\
\hline & PIVC insertion time (Min) & 2.6 & 3.5 & $+35 \%$ \\
\hline & First stick success rate $(\%)$ & 91.5 & 83.4 & $-9 \%$ \\
\hline & PIVC indwelling time (Days) & 3.7 & 2.1 & $-43 \%$ \\
\hline \multirow{6}{*}{$\begin{array}{l}\text { Catheter-related } \\
\text { Complications }\end{array}$} & Redness at insertion site $(\%)$ & 8.6 & 19.4 & $+126 \%$ \\
\hline & Swelling /complaints about pain (\%) & 7.5 & 18.2 & $+143 \%$ \\
\hline & Phlebitis (\%) & 6.3 & 16.0 & $+154 \%$ \\
\hline & Catheter displacement (\%) & 1.8 & 6.8 & $+278 \%$ \\
\hline & Extravasation $(\%)$ & 3.3 & 11.8 & $+258 \%$ \\
\hline & Catheter-related bloodstream infection (\%) & 0 & 0.03 & NA \\
\hline \multirow{2}{*}{ Product Training } & Number of training per year & 3.8 & 0.8 & $-79 \%$ \\
\hline & Duration of each training (Min) & 65.0 & 10.7 & $-84 \%$ \\
\hline
\end{tabular}

\subsection{PIVC Placement}

Compared with the MNC's PIVC, the local PIVC products had more challenges in PIVC placement (Table 3). The indwelling time of a PIVC reduced from 3.7 days to 2.1 days and the first stick success rate decreased from $91.5 \%$ to $83.4 \%$. Correspondingly, the average number of PIVCs used per patient increased from 2.0 to 3.5 and the mean insertion time increased by 0.9 minutes per PIVC insertion, thus indicating an increase in nurses' workloads.

Quotes on PIVC placement:

1) "We used to use 1 or 2 needles for the MNC's PIVC. Now with the new brand (local PIVC), it may take 3 or 4 needles to solve the problem (i.e. finish the treatment process)."
2) "Now (local PIVC) insertion takes a little more time than BD (Becton, Dickinson and Company) does, around 1-2 extra minutes."

3) "Now the first stick success rate is less than $70-80 \%$, which is definitely less than before. There was no problem for the MNC's (PIVC) to reach a success rate of over $90 \%$."

4) "Now the needles (local PIVC) stop working after 1-2 days. For BD needles, the indwelling time was around 3-4 days, and some can even reach 6-7 days."

\subsection{Catheter-related Complications}

The frequency of PIVC-related complications increased substantially with the transition from the MNC's PIVC to local PIVC (Table 3). The commonly reported complications 
such as occlusion before VoBP vs. after VoBP were $6.5 \%$ vs. $23.8 \%$, redness $8.6 \%$ vs. $19.4 \%$, swelling $7.5 \%$ vs. $18.2 \%$ and phlebitis $6.3 \%$ vs. $16.0 \%$, thus increasing by $266 \%$, $162 \%, 143 \%$ and $154 \%$, respectively due to product change. Furthermore, catheter displacement (1.8\% vs. $6.8 \%)$ and extravasation (3.3\% vs. $11.8 \%$ ) increased by $278 \%$, and $258 \%$, respectively after product change. CRBSI, a severe complication, was rarely found; the frequency was none for the MNC's PIVC and $0.03 \%$ for local brands' PIVC on average.

Quotes on complications:

1) "There were 2-3 cases (redness) per month with BD. Now there are 5-6 cases (local PIVC) per month."

2) "The MNC's product (PIVC) is still better. The patient said he didn't feel much about the insertion, and it did not hurt very much. However, with the new brand (local PIVC), after two days of insertion, the arm has an obvious feeling of swelling and a little painful."

3) "Now (using local PIVC) the frequency of phlebitis occurred is 5-6 cases per month. When we were using BD (MNC's PIVC), there were 2-3 cases per month."

4) "Now (using local PIVC) there are at least 10 cases (occlusion) per month. The condition used to be good with BD PIVC, I think there were not much, around 2-3 cases per month."

5) "Now (using local PIVC), catheter displacement happens around $30 \%$ of the time. For the BD brand, it did not have the tailing situation, so the occurrence was around $10 \%$."

6) "Now (using local PIVC), the occurrence rate of extravasation is as high as around $80-90 \%$. With the former BD brand (PIVC), unless the patient was aged, the young patients usually did not have this condition."

\subsection{Product Quality and Defects}

In terms of product quality, more than half of the nurses $(15 / 27)$ complained that local flimsy needles led to difficulties in PIVC insertion. A total of $48 \%$ of nurses (13/27) mentioned that the material of local PIVC was hard, which resulted in uncomfortable patient experiences. Product design issues, including the "improper length of needles" (5/27) and "long heparin caps" (4/27) were also reported by interviewees (Table 2). Nurses explained that long needles were inconvenient for single-hand operation of the device and long heparin caps increased blood return, resulting in occlusion.

Quotes on product issues:

1) "There is strong resistance (local PIVC) during the insertion, and this may be due to the blunt needle."

2) "BD (PIVC) is easy to rebound while other brands will have a crease and do not rebound. It is easy to block the catheter if patients move."

3) "During the indwelling period, the rear end of heparin cap (local PIVC) cracks and the liquids leak out."

4) "For the previous MNC's PIVC, I can connect it with one hand and finish with the other hand. But the current needle (local catheter) cannot be operated with one hand since the structure and design are not suitable."

5) "The pulley (local PIVC) doesn't slide very smoothly."

6) "The connection (local PIVC) may have some problems, and the leak will happen when there is a connection issue."

7) "This current local PIVCs doesn't feel as good as previous BD PIVCs, the steel needle and the soft needle of current local PIVC do not fit well."

8) "The heparin cap (local PIVC) is longer. If we flush the tube, the time will be longer."

\subsection{Product Training}

About half of nurses (13/27) mentioned that product training was important for improving their confidence in PIVC insertion and having better clinical operations, which is related to the daily work of nurses (Table 2). Manufacturers of high-quality product provided product training support and were available to solve issues, answer questions and re-train if needed. However, decreases were observed in both training frequency (from 3.8 times/year with the $\mathrm{MNC}$ to 0.8 times/year with local manufacturers) and duration (65 mins/session with the MNC to $10.7 \mathrm{mins} / \mathrm{session}$ with local manufacturers) after the product change, indicating the training problems for local brands (Table 3).

Quotes on product training:

1) "They (the trainer from local catheter manufacturers) just opened the samples and briefly demonstrated how to use them, which took less than five minutes, and then they left. We have no chance to provide feedback or ask questions as we do not have their contact information."

2) "The angle of puncture, the means to use the tube and other information including catheter maintenance are learned from BD's training sessions."

3) "After getting familiar with the operation process, you will not be nervous about handling patients. The patients will also trust you."

4) "The training helped us to improve the puncture success rate by at least $30 \%$ after changing the way we puncture."

5) "Training helped us to reduce the time for puncture from $1.5 \mathrm{~min}$ to $1 \mathrm{~min}$, including sealing."

6) "Through training, we (nurses) could learn certain techniques to effectively reduce pain."

\section{Discussion}

To the best of our knowledge, this is the first exploring the clinical impact of different PIVC products under the background of VoBP in China from clinical perspectives. Overall, the results from clinical perspective suggested that compared with the MNC's PIVC before VoBP, there are more challenges in PIVC placement, increased occurrence of complications, more product quality and design issues, and reduced manufacturer-led training with local PIVC after VoBP. Although there are currently no standard criteria to evaluate PIVC performance, indwelling time, complication rates, product quality and product training have been widely 
used to evaluate PIVCs in previous research and clinical practice [26-28].

Indwelling time is often used as a clinical indicator to demonstrate PIVC's efficiency. Our results demonstrated that after product change, PIVC indwelling time decreased from 3.5 days to 2.1 days. According to the recommendation from Chinese insertion guidelines, PIVCs should be changed every 72-96 hours (3-4 days) to reduce the risk of PIVC failure [29] The performance of the MNC's PIVC met the range of indwelling time recommended in the guideline but the indwelling time of local brands' PIVCs was lower, indicating lower PIVC efficiency. The shortened indwelling time also resulted in the increase in the number of PIVCs used and added to nurses' workloads, leading to extra medical resource consumption for routine catheter insertion, care, and maintenance.

PIVC related complications can be considered as a metric to evaluate PIVC quality. After the product change due to the implementation of VoBP, catheter-related complications such as occlusion, redness, swelling, phlebitis, extravasation and dislodgement increased notably, which may require removal of the existing PIVC and replacing it with a new PIVC or corresponding treatments [30]. Additionally, patients may experience discomfort and have complaints due to increased complications [15]. Therefore, the occurrence of complications should be regarded as another important indicator of a PIVC's performance.

Along with clinical indicators, a PIVC's design and materials should also be considered. Previous research demonstrated that complication rates varied by PIVC design and materials whereas the insertion technique of different PIVCs were similar [31]. For example, the MNC's PIVC with Vialon material showed better flexibility than those of local brands with Teflon material. The Vialon catheter resulted in less phlebitis and a lower risk of phlebitis during the period 49 to 72 hours after the insertion than the Teflon one [32, 33]. Also, Vialon cannulae was associated with a $46 \%$ lower incidence than the Teflon type in terms of infusion thrombophlebitis [34]. On top of this, the nurses complained that flimsy or lengthy needles and hard catheter materials were associated with difficulties in insertion. Given the relationship between product design and PIVC's clinical performance, specific standards for PIVC's design should be set up.

Product training could be another important consideration when the authority made decisions between PIVC's manufactures. During interviews, nurses reported the product training including insertion, maintaining, and Q\&A session is helpful in improving nurses' insertion skills and optimizing PIVC placement outcomes. A previously published study supported that training on PIVC insertions improved junior nurses' PIVC knowledge and operation [35]. Apart from skills, literature also reported that training significantly improved junior nurses' confidence in PIVC insertion [36]. Therefore, for products such as PIVCs where nurses' knowledge, confidence and skill level influence patient outcomes, it is important that manufacturers work closely with the nurses to provide sufficient hands-on training and education.
In addition to the above-mentioned indicators, appropriate indwelling needles should be selected according to different blood vessels. Special considerations should be taken for pediatric patients who are more vulnerable to complications owing to defective products. For example, neonates have fragile venous access, and therefore, multiple insertions can deplete their venous access points [37-39]. In this way, it is suggested that pediatric departments should be granted with more flexibility in PIVC purchasing. Similarly, elderly patients also have fragile and difficult-to-access veins [37]. Thus, it would be beneficial to use PIVCs with higher first stick success rates, longer dwell times and lower complication rates among these patients, that is, the procurement of higher-quality indwelling needles should be prioritized.

This study sheds light on the clinical performance of different PIVC products used before and after VoBP, indicating the importance of considering clinical impacts of PIVCs and then improving the current policy. The implementation of VoBP has led to significant benefits to patients' accessibility for cost-effective drugs [40], which indicated the direction of future medical products purchasing in China [40]. Nevertheless, VoBP of medical consumables such as PIVC is still at an early stage, and proper adjustments or improvements will be beneficial for the scale-up of VoBP. After understanding the VoBP-derived challenges that healthcare workers are confronting, policymakers are suggested to develop standard and quantifiable criteria to assess the quality consistency and performance of the medical consumables. In addition, product monitoring systems should be set up to ensure product quality and optimize patients' long-term health outcomes.

As the first evidence highlighting the outcomes associated with different PIVCs under the implementation of VoBP, the strengths of this study include the adoption of both qualitative and quantitative methods to understand nurses' perspectives in clinical settings. As primary users of PIVCs, nurses can bring valuable insights on product issues, clinical placement, complications as well as their experience in training provided by manufacturers. After low-value medical consumables were listed in the scope of VoBP, there is no public evidence analyzing clinical outcomes of products used before and after the policy. Therefore, this study addressing this issue at the early stage of policy implementation could provide important evidence for policy optimization. Besides, previous research on PIVC use focused on quantitative results [41-43] and ignored nurses' experiences which is also considered to be critical. This study applied both qualitative and quantitative analyses; a qualitative method comprehensively explored different domains influenced by product change, and a quantitative analysis helped to understand the variation in each indicator.

There are two limitations to be considered in this study. One is the sample size issue; the interviewee recruitment was affected by the COVID-19 pandemic. The nurses' schedule was majorly occupied during the interview months of March and April, since their colleagues may be appointed to support 
Wuhan or other COVID-19 pandemic areas. Additionally, most of the participants were from tertiary hospitals $(25 / 27)$ and internal medicine (24/27). Although saturation was reached, the study results should still be cautiously interpreted and applied to other hospital tiers and departments. The second limitation is the recall bias issue since the data was collected retrospectively via interviews. Collecting responses from nurses both before and after the implementation of VoBP may avoid some recall bias, however, it was difficult to predict when the VoBP would be implemented and in which province/city beforehand. Acknowledging the intrinsic limitation of recall bias, trained and experienced interviewers and a well-designed discussion guide were utilized to minimize the impact of recall bias, and all participants were encouraged to summarize key data \& information prior to the interview to ensure the data accuracy. Future studies with larger and more representative interviewees and prospective data collection are warranted to constantly update our understanding of the clinical use of different PIVCs with the implementation of VoBP in China.

\section{Conclusion}

From clinical perspectives, following the implementation of VoBP and concomitant PIVC product change, more challenges in clinical placement, increased complication rates, more product quality issues, and reduced manufacturer-led training were recognized in clinical settings with local PIVCs compared to the MNC's PIVCs. The distinct clinical impacts of different PIVC products used before and after VoBP indicated the importance of considering product performance and monitoring product quality in VoBP process apart from price competition.

The VoBP policy adopted by China government has brought significant benefits to lower patients' economic burden, guiding the future direction of medical products purchasing in China. However, the VoBP of medical consumables such as PIVCs is still at an early stage, and proper adjustments will be beneficial for the scale-up of VoBP. Before we reach a more comprehensive VoBP process, clear evaluation standards on clinical performance of PIVCs are warranted to ensure patients' long-term health outcomes. Furthermore, policymakers are suggested to establish quality evaluation criteria and strengthen product monitoring mechanism for VoBP policy optimization in China.

\section{Source of Financial Support}

This project was sponsored by Becton, Dickinson and Company, NJ, US. Funding was not contingent upon publication of the manuscript. The authors have no other relevant affiliations or financial involvement with any organization or entity.

\section{Conflict of Interest}

All the authors do not have any possible conflicts of interest.

\section{Availability of Data and Materials}

Data are not publicly available, but the authors are willing to provide additional data upon request.

\section{References}

[1] Alexandrou E, Ray-Barruel G, Carr PJ, et al. Use of Short Peripheral Intravenous Catheters: Characteristics, Management, and Outcomes Worldwide. J Hosp Med. 2018; 13 (5).

[2] Wei T, Li XY, Yue ZP, et al. Catheter dwell time and risk of catheter failure in adult patients with peripheral venous catheters. J Clin Nurs. 2019; 28 (23-24): 4488-95.

[3] The Royal Children's Hospital Melbourne, Clinical Guidelines: Peripheral intravenous (IV) device management. https://www.rch.org.au/rchcpg/hospital_clinical_guideline_ind ex/Peripheral_Intravenous_IV_Device_Management/. Accessed Maȳ 26, 2020.

[4] Sabri A, Szalas J, Holmes KS, et al. Failed attempts and improvement strategies in peripheral intravenous catheterization. Biomed Mater Eng. 2013; 23 (1-2): 93-108.

[5] Sun H, Wang L, Guan X, et al. A survey of the status of infusion therapy in tertiary hospitals in China. Chinese Journal of Nursing. 2014; 49 (10): 1232-7.

[6] Burton, Patrick. "4+7: China's New Volume-Based Purchasing Policy." Pharma Boardroom, pharmaboardroom.com/articles/4-plus-7-chinas-new-volumebased-purchasing-policy/.

[7] Yue, Xiao. "4+7" Drug Procurement Reform in China. China National Health Development Research Center, 2019.

[8] The State Council of the People's Republic of China. Reform plan for managing high-value medical consumables. (2019) http://www.gov.cn/zhengce/content/2019-

07/31/content_5417518.htm. Accessed May 26, 2020.

[9] UNAIDS, Global HIV \& AIDS statistics - 2020 fact sheet. https://www.unaids.org/en/resources/fact-sheet. Accessed Nov 1 st 2020.

[10] Portal, M. R. "Peripheral intravenous (IV) device management." R Child.

[11] Tang M, He J, Chen M, et al. "4+7" city drug volume-based purchasing and using pilot program in China and its impact. Drug Discov Ther. 2019; 13 (6): 365-9.

[12] Abolfotouh MA, Salam M, Bani-Mustafa A, et al. Prospective study of incidence and predictors of peripheral intravenous catheter-induced complications. Ther Clin Risk Manag. 2014; 10: $993-1001$.

[13] Gao ZL, Guo L, Li LZ, Comparison on the clinical outcome and cost-effectivemenss analysis of perirpheral intravenous catheter vs wing needle set in infusion therapy. China Journal of Pharmaceutical Economics. 2018 (02).

[14] Cai Y, Zhu M, Sun W, et al. Study on the cost attributable to central venous catheter-related bloodstream infection and its influencing factors in a tertiary hospital in China. Health and quality of life outcomes. 2018; 16 (1): 1-6. 
[15] Kaur P, Rickard C, Domer GS, et al. Dangers of Peripheral Intravenous Catheterization: The Forgotten Tourniquet and Other Patient Safety Considerations. Vignettes in Patient Safety-Volume 4: IntechOpen; 2019.

[16] Palinkas L A, Horwitz S M, Green C A, et al. Purposeful sampling for qualitative data collection and analysis in mixed method implementation research [J]. Administration and policy in mental health and mental health services research, 2015, 42 (5): 533-544.

[17] Li ML. Observation on the effect of bedside ward round on improving nursing ability. Contemporary Nurse: Specialist Edition, 2017 (8): p. 168-170.

[18] Nelson J. Using conceptual depth criteria: addressing the challenge of reaching saturation in qualitative research. Qualitative research. 2017; 17 (5): 554-70.

[19] Walker JL. The use of saturation in qualitative research. Can J Cardiovasc Nurs. 2012; 22 (2): 37-46.

[20] Guest G, Bunce A, Johnson L. How many interviews are enough? An experiment with data saturation and variability. Field methods. 2006; 18 (1): 59-82.

[21] Bowen GA. Naturalistic inquiry and the saturation concept: a research note. Qualitative research. 2008; 8 (1): 137-52.

[22] Kerr C, Nixon A, Wild D. Assessing and demonstrating data saturation in qualitative inquiry supporting patient-reported outcomes research. Expert review of pharmacoeconomics \& outcomes research. 2010; 10 (3): 269-81.

[23] Turner-Bowker DM, Lamoureux RE, Stokes J, et al. Informing a priori sample size estimation in qualitative concept elicitation interview studies for clinical outcome assessment instrument development. Value in Health. 2018; 21 (7): 839-42.

[24] Nagpal K, Arora S, Vats A, et al. Failures in communication and information transfer across the surgical care pathway: interview study. BMJ Qual Saf. 2012; 21 (10): 843-9.

[25] Cutcliffe JR. Methodological issues in grounded theory. Journal of advanced nursing. 2000; 31 (6): 1476-84.

[26] Vinograd AM, Zorc JJ, Dean AJ, et al. First-attempt success, longevity, and complication rates of ultrasound-guided peripheral intravenous catheters in children. Pediatric emergency care. 2018; 34 (6): 376-80.

[27] Yuan H, Chen LF, Chen YJ, et al. Effect comparison of three kinds of venous indwelling needles in adult inpatients. Journal of Nursing (China). 25.8 (2018): 51-54.

[28] Legemaat M, Carr PJ, van Rens RM, et al. Peripheral intravenous cannulation: complication rates in the neonatal population: a multicenter observational study. The journal of vascular access. 2016; 17 (4): 360-5.

[29] China National Health and Family Planning Commission. Nursing practice standards of intravenous therapy. 2014.
[30] Jones RK. Short Peripheral Catheter Quality and Economics: The Intravenous Quotient. Journal of Infusion Nursing. 2018; 41 (6): 365-71.

[31] Chen J, Yu SN, Wang, CL, et al. Clinical application of 3 types of venous indwelling needles. Journal of Guangdong Medical University. 01: 106-108. (2019).

[32] Jacquot C, Fauvage B, Bru J, et al., editors. Effect of type of material on thrombophlebitis risk with peripheral venous catheters. ANNALES FRANCAISES D ANESTHESIE ET DE REANIMATION; 1989: EDITIONS SCIENTIFIQUES ELSEVIER 141 RUE JAVEL, 75747 PARIS CEDEX 15, FRANCE.

[33] Karadag A, Görgülü S. Effect of two different short peripheral catheter materials on phlebitis development. Journal of Infusion Nursing. 2000; 23 (3): 158-66.

[34] Maki DG, Ringer M. Risk factors for infusion-related phlebitis with small peripheral venous catheters: a randomized controlled trial. Annals of internal medicine. 1991; 114 (10): 845-54.

[35] Yang CR, Huang XL, Chen MY, et al. Training of intravenous catheter system to junior nurses based on clinical nursing ability. Modern Clinical Nursing. 08: 59-64 (2018).

[36] Keleekai NL, Schuster CA, Murray CL, et al. Improving nurses' peripheral intravenous catheter insertion knowledge, confidence, and skills using a simulation-based blended learning program: a randomized trial. Simulation in Healthcare. 2016; 11 (6): 376.

[37] Armenteros-Yeguas V, Gárate-Echenique L, Tomás-López MA, et al. Prevalence of difficult venous access and associated risk factors in highly complex hospitalised patients. Journal of clinical nursing. 2017; 26 (23-24): 4267-75.

[38] Doniger SJ, Ishimine P, Fox JC, et al. Randomized controlled trial of ultrasound-guided peripheral intravenous catheter placement versus traditional techniques in difficult-access pediatric patients. Pediatric emergency care. 2009; 25 (3): 154-9.

[39] Naik VM, Mantha SSP, Rayani BK. Vascular access in children. Indian J Anaesth. 2019; 63 (9): 737-45.

[40] $\mathrm{Hu}$ SL. Analysis on economics theoretical basis and impact in drug bulk purchasing with quantity. Soft Science of Health 01: 3-5 (2019).

[41] Wei T, Tan P, Chen YY, et al. Survey of the use of peripheral venous catheters in adult patient. Journal of Nursing Science. 17 (2018): 4.

[42] Zhang Y, Chen L. Analysis on the Application status and existing problems of intravenous indwelling needle in our hospital. Journal of Disease Monitor and Control. 7: 32 (2014).

[43] Huang P, Liu LP, Wang L. Analysis of Current Status and Influencing Factors of Venous Indwelling Needles in Hospitals of Grade two and Above in Chongqing. Journal of Qilu Nursing, 23.14 (2017): 3-5. 